\title{
A LATtice analogue OF A THEOREM OF CHOW
}

\author{
Dedicated to the memory of Hanna Neumann
}

\section{GEORGE MAXWELL}

(Received 22 May 1972)

Communicated by M. F. Newman

Let $K$ be a skewfield, $E$ a left vector space over $K, r$ an integer $\geqq 1$ and $G_{r}(E)$ the set of all $r$-dimensional subspaces of $E$, called the Grassmannian of index $r$. The function $d(A, B)=r-\operatorname{dim}(A \cap B)$ is a distance on $G_{r}(E)$. If $K^{\prime}$ is a skewfield and $E^{\prime}$ a left vector space over $K^{\prime}$, then any semilinear isomorphism $u: E \rightarrow E^{\prime}$ (relative to an isomorphism $K \rightarrow K^{\prime}$ ) induces a distance preserving bijection $G_{r}(u): G_{r}(E) \rightarrow G_{r}\left(E^{\prime}\right)$. When $E$ has finite dimension $n$ and $2 r=n$, another example of such a mapping is obtained by taking $K^{\prime}=K^{o p}, E^{\prime}=E^{*}$ and defining $w_{r}: G_{r}(E) \rightarrow G_{r}\left(E^{*}\right)$ to be $w_{r}(A)=\left\{f \in E^{*} \mid f(A)=0\right\}$.

In 1949, Chow [2] proved the following converse:

THEOREM. Suppose $E$ and $E^{\prime}$ both have finite dimension $n$ and $2 \leqq r \leqq n-2$. Let $\phi$ be a distance preserving bijection $G_{r}(E) \rightarrow G_{r}\left(E^{\prime}\right)$. Then if $2 r \neq n$ there exists a semilinear isomorphism $u: E \rightarrow E^{\prime}$ such that $\phi=G_{r}(u)$, while in the case $2 r=n, \phi$ may also be of the form $G_{r}(v) w_{r}$, where $v$ is a semilinear isomorphism $E^{*} \rightarrow E^{\prime}$.

The proof consists of a reduction to the case $r=1$, followed by an application of the fundamental theorem of projective geometry. The purpose of this note is to place the first part of Chow's argument in a lattice-theoretic setting and apply it to some other examples.

Let $L$ be a lattice with 0 ; we assume that $L$ is discrete i.e. that for all $a, b \in L$, the supremum of lengths of chains from $a$ to $b$ is finite. Such a lattice has a height function $h: L \rightarrow \mathbb{N}$, defined by $h(a)=$ sup. of lengths of chains from 0 to $a$. The supremum of all numbers $h(a)$, which may be infinite, is denoted by $h(L)$. We shall assume furthermore that $L$ is atomic and modular; the height function then satisfies the identity

$$
h(a \vee b)+h(a \wedge b)=h(a)+h(b) .
$$


For every integer $r \geqq 1$, the set $L_{r}$ of all elements of height $r$ in $L$ is a metric space under the distance $d(a, b)=r-h(a \wedge b)$.

Atoms $p, q, r \in L_{1}$ are called collinear if they are all contained in some element of $L_{2}$. If $L^{\prime}$ is a lattice with the same properties as $L$, a bijective mapping $u: L_{1} \rightarrow L_{1}^{\prime}$ is called a collineation provided that atoms $p, q, r \in L_{1}$ are collinear if and only if their images in $L_{1}^{\prime}$ are collinear.

Lemma 1. A collineation $u: L_{1} \rightarrow L_{1}^{\prime}$ induces a distance preserving bijection $u_{r}: L_{r} \rightarrow L_{r}^{\prime}$ by defining $u_{r}(a)=u\left(p_{1}\right) \vee \cdots \vee u\left(p_{r}\right)$, where $a=p_{1} \vee \cdots \vee p_{r}$ is some representation of $a$ as a join of atoms.

Granted this lemma, our principal result can be stated as follows:

THEOREM. Suppose $L$ and $L^{\prime}$ are discrete atomic modular lattices such that $h(L)=h\left(L^{\prime}\right)$ and $2 \leqq r \leqq h(L)-2$. Let $\phi$ be a distance preserving bijection $L_{r} \rightarrow L_{r}^{\prime}$. Then if $2 r \neq h(L)$ there exists a collineation $u: L_{1} \rightarrow L_{1}^{\prime}$ such that $\phi=u_{r}$, while in the case $2 r=h(L), \phi$ may also be of the form $w v_{r}$, where $w$ is a particular exceptional mapping $L_{r} \rightarrow L_{r}^{\prime}$ and $v$ is a collineation $L_{1} \rightarrow L_{1}$.

Taking $L\left(L^{\prime}\right)$ to be the lattice of all finite dimensional subspaces of $E\left(E^{\prime}\right)$, one recovers essentially the theorem of Chow, with the added observation that it remains valid for infinite dimensional spaces (in an even simpler form). Another interesting example is obtained when $L$ is the lattice $F(S)$ of all finite subsets of a set $S$; in this case, the theorem simplifies to read as follows:

Corollary. If $S$ and $S^{\prime}$ are sets, any distance preserving bijection $\phi: F_{r}(S) \rightarrow F_{r}\left(S^{\prime}\right)$ is induced by a bijection $u: S \rightarrow S^{\prime}$ unless both $S$ and $S^{\prime}$ are finite of cardinality $n$, and $2 r=n$, when $\phi$ may also be of the form $\phi(A)$ $=u(S \backslash A)$.

It is curious to note that, just as the fundamental theorem of projective geometry is used $\left[3\right.$, p. 93] in determining the automorphisms of $G L_{n}(K)$, so can a special case of the above corollary be applied to the automorphisms of finite or infinite symmetric groups $S_{X}$. Indeed, one first shows by some means that for $\operatorname{card}(X) \neq 6$, the set of transpositions is left invariant by an automorphism and then remarks that the distance between transpositions (regarded as elements of $\left.F_{2}(X)\right)$ is 0,1 or 2 according to whether the order of their product is 1,3 or 2 . Since automorphisms preserve order, the corollary shows that for $\operatorname{card}(X) \neq 4$ the automorphism is induced, at least on transpositions, by conjugation with an element $u \in S_{X}$. The argument is then concluded as in [4].

Finally, we remark that if $L$ satisfies $h(L)<\infty$ in addition to the other hypotheses, a theorem of Birkhoff [1, p. 93] says that $L$ must be a product of a boolean algebra with projective geometries. In this case, Chow's theorem covers the simple Desarguean factors, while the corollary above takes care of the boolean algebra. 
We now turn to the proofs. Elements $a, b \in L_{r}$ are called adjacent if $d(a, b)=1$.

LeMMA 2. If $d(a, b)=k$, there exists a sequence $c_{0}, \cdots, c_{k}$ of elements in $L_{r}$ such that $c_{0}=0, c_{k}=b$ and $c_{i-1}$ is adjacent to $c_{i}$ for $1 \leqq i \leqq k$.

ProOF. An argument of Birkhoff $[1$, p. 88] shows that a lattice satisfying our assumptions is relatively complemented. Therefore $a=(a \wedge b) \vee x$, $b=(a \wedge b) \vee y$ for some $x$ and $y$ disjoint from $a \wedge b$ and from each other. If $x=p \vee x^{\prime}, y=q \vee y^{\prime}$ for some atoms $p$ and $q$, the element $c_{k-1}=(a \wedge b) \vee p$ $\vee y^{\prime}$ satisfies $d\left(a, c_{k-1}\right)=k-1$ and $d\left(c_{k-1}, b\right)=1$; the argument is now concluded by induction.

Lemma 3. A bijection $\phi: L_{r} \rightarrow L_{r}^{\prime}$ preserves distance if and only if both $\phi$ and $\phi^{-1}$ preserve adjacence.

Proof. Suppose $d(a, b)=k$. Then, with the notation of lemma 2, the sequence $\phi\left(c_{0}\right), \cdots, \phi\left(c_{k}\right)$ is such $\phi\left(c_{i-1}\right)$ and $\phi\left(c_{i}\right)$ are adjacent for $1 \leqq i \leqq k$. The triangle inequality implies $d(\phi(a), \phi(b)) \leqq d(a, b)$. (Actually, this inequality in turn implies that $\phi$ preserves adjacence, for if $d(a, b)=1, d(\phi(a), \phi(b))$ cannot be 0 since $\phi$ is injective and must therefore be 1.) Since $\phi^{-1}$ also preserves adjacence, we conclude that $d\left(\phi^{-1}(a), \phi^{-1}(b)\right) \leqq d(a, b)$ and together the two inequalities imply that $\phi$ preserves distance.

PROOF OF LEMMA 1. If $a \in L_{r}$ is written as a join $p_{1} \vee \cdots \vee p_{r}$ of $r$ atoms, the latter are necessarily independent. We show by induction that an atom $q \leqq p \vee \cdots \vee p_{r}$ if and only if $u(q) \leqq u\left(p_{1}\right) \vee \cdots \vee u\left(p_{r}\right)$. This is clear if $q=p_{r}$ or if $q \leqq p_{1} \vee \cdots \vee p_{r-1}$; otherwise, $q \vee p_{r}$ intersects $p_{1} \vee \cdots \vee p_{r-1}$ in an atom $t$. We must have $q \leqq q \vee p_{r}=t \vee p_{r}$ and thus $u(q) \leqq u(t) \vee u\left(p_{r}\right)$ since $u$ is a collineation; however, the induction assumption shows that $u(t) \leqq u\left(p_{1}\right) \vee \cdots$ $\vee u\left(p_{r-1}\right)$. The converse follows by applying the same argument to $u^{-1}$. We conclude in particular that the atoms $u\left(p_{1}\right), \cdots, u\left(p_{r}\right)$ are independent so that $u\left(p_{1}\right) \vee \cdots \vee u\left(p_{r}\right) \in L_{r}^{\prime}$; secondly, if $p_{1}^{\prime} \vee \cdots \vee p_{r}^{\prime}$ is another representation of a as a join of atoms, $u\left(p_{i}\right) \leqq u\left(p_{1}^{\prime}\right) \vee \cdots \vee u\left(p_{r}\right)$ so that $u\left(p_{1}^{\prime}\right) \vee \cdots \vee u\left(p_{r}^{\prime}\right)=u\left(p_{1}\right)$ $\vee \cdots \vee u\left(p_{r}\right)$. Thus $u_{r}$ is well-defined, with inverse $\left(u^{-1}\right)_{r}$. If $a, b \in L_{r}$ are adjacent then, since $u_{r}(a)$ and $u_{r}(b)$ clearly contain $u_{r-1}(a \wedge b)$, they must be adjacent or equal, but the latter is impossible. We conclude by lemma 3 that $u_{r}$ preserves distance.

We now come the the theorem itself. The first step is to determine the structure of a maximal set $M$ of pairwise adjacent elements of $L_{k}$. Since $k<h(L), M$ must have at least two elements $a$ and $b$. If there exists some $c \in M$ which does not contain $a \wedge b$, then every element of $M$ is contained in $a \vee b$. Indeed, if $d \in M$ does not contain $a \wedge b$, then $a \wedge d \neq b \wedge d$ so that $d=(a \wedge d) \vee(b \wedge d)$ $\leqq(a \vee b) \wedge d$ i.e. $d \leqq a \vee b$. If, on the other hand, $d \in M$ does contain $a \wedge b$, then $c \wedge d$ must be distinct from $a \wedge d=a \wedge b$ so that $d=(c \wedge d) \vee(a \wedge d)$ 
$\leqq(c \vee a) \wedge d=(a \vee b) \wedge d$ and again $d \leqq a \vee b$. This argument shows that $M$ is either of the form $C_{x}=\left\{a \in L_{r} \mid a \geqq x\right\}$ for some $x \in L_{r-1}$ or $C^{y}=\left\{a \in L_{r} \mid a \leqq y\right\}$ for some $y \in L_{r+1}$, since such sets consist of pairwise adjacent elements. If a set $C_{x}$ is not maximal, it is contained in another set of the form $C_{y}$ or $C^{y}$. The first alternative is clearly impossible and the second is ruled out by the condition $r \leqq h(L)-2$. Indeed, if $p$ is an atom not contained in $y$, then $p \vee x \in C_{x} \mid C^{y}$. Similarly, the condition $r \geqq 2$ shows that sets of the form $C^{y}$ are maximal.

Now suppose that $\phi: L_{r} \rightarrow L_{r}^{\prime}$ is a distance preserving bijection. If $x \in L_{r-1}$, $\phi\left(C_{x}\right)$ is a maximal set of pairwise adjacent elements and thus of the form $C_{y}$ or $C^{y}$. If the first alternative holds for a particular $x \in L_{r-1}$, then it holds for all elements of $L_{r-1}$. To see this, it suffices to remark that if $x \neq x^{\prime}, C_{x} \cap C_{x}$, consists of at most one element $\left(x \vee x^{\prime}\right)$, while an intersection of a set $C_{y}$ with a set $C^{y^{\prime}}$ has at least two elements, namely $y \vee p$ and $y \vee q$, where $p$ and $q$ are distinct atoms contained in $y^{\prime}$ but not in $y$.

It follows that $\phi$ induces either a bijection $\psi: L_{r-1} \rightarrow L_{r-1}^{\prime}$ or $L_{r-1} \rightarrow L_{r+1}^{\prime}$. Since $x$ and $x^{\prime}$ are adjacent precisely when $C_{x} \cap C_{x^{\prime}} \neq \varnothing$ or $C^{x} \cap C^{x^{\prime}} \neq \varnothing$, it follows that both $\psi$ and $\psi^{-1}$ preserve adjacence and hence distance. Unless $h(L)$ $<\infty$ and $2 r=h(L)$, the second alternative is impossible. If $2 r<h(L)$, the distance between elements of $L_{r-1}$ is at most $r-1$, while the distance between elements $L_{r+1}^{\prime}$ can be as high as $r$. If $2 r>h(L)$, the second distance is at most $h(L)-r-1$, while the first can be $h(L)-r$.

The original mapping $\phi$ can be recovered from $\psi$ through the equation $\psi\left(C^{x}\right)$ $=C^{\phi(x)}$, which also shows that even if $2(r-1)$ happens to be $h(L), \psi$ still maps sets of the form $C_{x}$ to sets of the same form and thus induces a mapping $L_{r-2}$ $\rightarrow L_{r-2}^{\prime}$. Eventually, we obtain a collineation $u: L_{1} \rightarrow L_{1}^{\prime}$ such that $\phi=u_{r}$.

If there does exist, when $2 r=h(L)$, an exceptional mapping $w$ which induces a mapping $L_{r-1} \rightarrow L_{r+1}^{\prime}$ then for every other such mapping $\phi, w^{-1} \phi$ induces a mapping $L_{r-1} \rightarrow L_{r-1}$ and thus comes from a collineation $v: L_{1} \rightarrow L_{1}$. This completes the proof.

\section{References}

[1] G. Birkhoff, Lattice Theory, (3rd edition Colloq. Publ. vol. 25, Amer. Math. Soc., 1967).

[2] W. L. Chow, 'On the geometry of algebraic homogeneous spaces', Annals of Math. 50 (1949), 32-67.

[3] J. Dieudonné, La géométrie des groupes classiques, (3ème edition Springer Verlag, Berlin, 1971).

[4] J. Schreier and S. Ulam, 'Über die Automorphismengruppe der Permutationsgruppe der natürlichen Zahlenfolge', Fund. Math. 28 (1937), 258-260.

Department of Mathematics,

University of British Columbia,

Vancouver 8, B. C. Canada. 